\title{
INVESTIGACIONES
}

\section{Los proyectos como contextualizadores de nociones básicas de estadística y probabilidad en la formación inicial de profesores de educación primaria*}

\author{
Using project-work to contextualize basic statistic and probabilistic notions \\ in the initial training of primary school teachers
}

\author{
Hernán Rivas Catricheo ${ }^{a}$, Juan D. Godino ${ }^{b}$, Pedro Arteaga Cezón ${ }^{c}$ \\ a Pontificia Universidad Católica de Chile, Campus Villarrica \\ Correo electrónico: hrivasa@uc.cl \\ b Universidad de Granada, Granada, España \\ Correo electrónico: jgodino@ugr.es \\ c Universidad de Granada, Granada, España \\ Correo electrónico: parteaga@ugr.es
}

\begin{abstract}
RESUMEN
El objetivo de este trabajo es analizar y evaluar un proceso formativo, sobre nociones básicas de estadística y probabilidad, llevado a cabo con una muestra de 58 futuros profesores de educación primaria. Aplicando herramientas teóricas del enfoque ontosemiótico del conocimiento y la instrucción matemáticos se analizan los tres proyectos de análisis de datos utilizados y la trayectoria didáctica implementada. Los hechos didácticos significativos identificados permiten evaluar la idoneidad didáctica del proceso, teniendo en cuenta las dimensiones epistémica, ecológica, cognitiva, afectiva, interaccional y mediacional. Se muestra cómo el uso de situacionesproblemas, optimiza el aprendizaje de la estadística, al dar sentido a las técnicas y teorías matemáticas, aunque la gestión del proceso de estudio requiere que el profesor recuerde los conocimientos previos necesarios en distintos momentos del proceso de estudio y sistematice finalmente los conocimientos pretendidos.
\end{abstract}

Palabras clave: formación docente, trayectoria didáctica, idoneidad didáctica

\section{ABSTRACT}

In this paper we analyze and evaluate a training process on basic statistics and probability, conducted with a sample of 58 future teachers of primary school teachers. Applying theoretical tools from the onto-semiotic approach to mathematical knowledge and instruction the three data analysis projects used, as well as the implemented didactic trajectory are analyzed. The significant didactical facts identified allow assessing the didactical suitability of the process, taking into account the epistemic, ecological, cognitive, emotional, interactional and mediational dimensions. The results suggest that the use of situations - problems, optimizes the learning of statistics, in making sense of the mathematical techniques and theories; however, the management of the study process requires the teacher to revisit the background knowledge needed at different phases of the study process and to finally systematize the intended knowledge.

Key words: teacher training, didactical trajectory, didactical suitability

Esta investigación ha sido realizada en el marco de los proyectos de investigación EDU2012-31869, y EDU2013-41141-P (Ministerio de Economía y Competitividad, España), y del Programa de Capital Humano Avanzado de la Comisión Nacional Científica y Tecnológica (CONICYT) de Chile. 


\section{INTRODUCCIÓN}

La estadística es una de las áreas de la matemática escolar cuya enseñanza ha experimentado un desarrollo importante durante los últimos años. Estos contenidos han sido incorporados de forma generalizada al currículo de matemáticas de la enseñanza primaria y secundaria en la mayoría de los países desarrollados, como así también en diferentes países en vías de desarrollo. A nivel internacional, la propuesta curricular sobre los Principios y Estándares para la Educación Matemática del National Council of Teachers of Mathematics (NCTM, 2000, 2014) incluye, dentro de los estándares de contenido, el estándar Análisis de datos y Probabilidad, en el cual se propone un conjunto de capacidades que los programas de enseñanza de todas las etapas deberían contemplar. Por otra parte, las Guidelines for Assessment and Instruction in Statistics Education (GAISE) proporcionan un marco conceptual para la enseñanza de la estadística y la probabilidad en la etapa Pre-K-12 (Infantil hasta Secundaria), centrado en la resolución de problemas de análisis de datos que promueven distintos niveles de comprensión (Franklin et al., 2005). En España, los actuales diseños curriculares para la enseñanza obligatoria (MEC, 2006; MECD, 2014) incluyen un bloque específico sobre estadística y probabilidad en todos los niveles de enseñanza, tanto de educación primaria como secundaria.

El desarrollo que ha experimentado la estadística en el ámbito escolar requiere que los profesores reciban una formación adecuada. Este desafío ha sido reconocido y asumido en investigaciones enfocadas en analizar la problemática de formación del profesorado en estadística y su didáctica (Batanero, Burrill y Reading, 2011). Entre estas investigaciones hay trabajos que aportan orientaciones sobre aspectos metodológicodidácticos para la formación estadística de los profesores, una de ellas es la metodología de trabajo con proyectos de análisis de datos (Batanero y Díaz, 2004). Esta forma de trabajo apunta a contextualizar los contenidos estadísticos a través de las diferentes fases de una investigación: formulación de un problema, decisión sobre los datos a recoger, análisis de datos, obtención de conclusiones sobre el problema planteado y redacción del informe final.

En este trabajo, se evalúa la implementación de un proceso formativo en estadística y probabilidad con futuros profesores de educación primaria que estuvo centrado en la resolución de tres proyectos de análisis de datos.

\section{MARCO TEÓRICO}

Este trabajo se sustenta en el Enfoque Ontosemiótico (EOS) del conocimiento y la instrucción matemáticos de Godino y colaboradores (Godino, Batanero y Font, 2007). En particular, se utiliza la noción de Hecho Didáctico Significativo (HDS) (Godino, Rivas, Arteaga, Lasa y Wilhelmi, 2014) como punto de partida para describir la trayectoria didáctica implementada y una Guía para la Valoración de la Idoneidad Didáctica de Procesos de Formación Estadística (GVID-PFE) desarrollada en el marco del EOS (Rivas, 2014). Tanto la noción de HDS, interpretada desde el EOS, como la GVID-PFE se fundamentan en la noción de idoneidad didáctica (Godino, 2013), la cual se define como la articulación coherente y sistémica de seis dimensiones: epistémica, ecológica, cognitiva, afectiva, interaccional y mediacional. 
Las cuestiones específicas que abordamos se formulan en los siguientes términos:

¿Qué objetos y procesos presentes en las prácticas matemáticas institucionales son movilizados a través de las tareas propuestas?

¿Qué tipo de relaciones se establecen con elementos del entorno en que se realiza el proceso educativo?

¿Qué prácticas, objetos y procesos matemáticos ponen en juego los estudiantes para resolver las tareas propuestas? ¿Cuáles de estas prácticas, objetos y procesos matemáticos son válidos desde la perspectiva institucional?

¿En qué medida se tienen en cuenta aspectos afectivos (actitudes, emociones, creencias, valores) de los estudiantes con relación a los objetos y procesos matemáticos estudiados?

¿Qué interacciones didácticas son implementadas para promover los aprendizajes? ¿Cómo se gestionan los recursos técnicos y temporales para favorecer los aprendizajes?

A continuación, se describe la noción de HDS y se explica el desarrollo de guías para la valoración de la idoneidad didáctica, en particular la guía que aplicamos en esta investigación.

\subsection{NOCIÓN DE HECHO DIDÁCTICO SIGNIFICATIVO}

La noción de HDS ha emergido en el EOS como una herramienta de análisis complementaria a las nociones de configuración y trayectoria didáctica (Godino, Contreras, y Font, 2006). En Wilhelmi, Font y Godino (2005) se interpreta un hecho didáctico como cualquier acontecimiento que tiene un lugar y un tiempo en el devenir de los procesos de instrucción matemática y que, por alguna razón, se considera como una unidad (por ejemplo, resolver una ecuación en la pizarra). Los hechos que implican una cierta regularidad explicable en el marco de una teoría constituyen un fenómeno; pero también pueden carecer de esa regularidad, en cuyo caso se tiene un fenómeno singular (dan pie a teoremas de existencia y a contraejemplos). A partir de esta noción, Godino et al. (2014) introducen la noción de hecho didáctico significativo en los siguientes términos:

Un hecho didáctico es significativo (HDS) si las acciones o prácticas didácticas que lo componen desempeñan una función, o admiten una interpretación, en términos del objetivo instruccional pretendido. La significatividad se puede entender desde el punto de vista del docente, del estudiante, o bien desde un punto de vista institucional externo al sistema didáctico, es decir, del sujeto que ha realizado el estudio preliminar y el diseño instruccional (Godino et al., 2014, p. 7).

La identificación e interpretación de hechos didácticos significativos requiere de herramientas conceptuales que fundamenten un análisis adecuado. La teoría de la idoneidad didáctica, desarrollada en el EOS, es una de las herramientas aplicables en 
este sentido. Esta noción se define como un criterio sistémico y coherente compuesto por seis dimensiones o facetas: epistémica, ecológica, cognitiva, afectiva, interaccional y mediacional. A continuación, se emplean estas dimensiones para describir sucintamente los tipos de HDS que se consideran en este trabajo:

- Epistémico: hechos que reflejan el tipo de tareas (problemas), lenguajes, reglas (definiciones, proposiciones, procedimientos) y argumentos puestos en juego.

- Ecológico: hechos que dan cuenta de las conexiones del proceso de estudio con elementos del entorno en que se realiza (currículo, medio sociocultural y profesional, conexiones intra e interdisciplinares). Se tienen en cuenta la innovación y la formación en valores.

- Cognitivo: hechos que muestran las relaciones que se establecen durante el proceso de instrucción con los aprendizajes previos, las adaptaciones curriculares a las diferencias individuales y los aprendizajes alcanzados teniendo en cuenta eventuales conflictos.

- Afectivo: hechos que expresan la forma en que se recogen los intereses y necesidades de los estudiantes para implicarlos en el proceso de estudio.

- Interaccional: hechos que dan cuenta de la interacción entre el profesor y los estudiantes y de los estudiantes entre sí, para observar en qué medida dichas interacciones permiten identificar y resolver conflictos de significado.

- Mediacional: sucesos que reflejan la forma en que se integran los recursos materiales, la distribución del espacio físico, la organización de los estudiantes y la gestión del tiempo para la enseñanza.

\subsection{GUÍAS PARA LA VALORACIÓN DE LA IDONEIDAD DIDÁCTICA}

En los últimos años, dentro del EOS se ha venido teorizando el uso de guías con indicadores de idoneidad didáctica como herramientas que apoyan la reflexión sistemática sobre las facetas y componentes que intervienen en un proceso de instrucción matemática (Godino, 2013; Rivas, 2014). Estas guías sintetizan el sistema complejo de conocimientos matemáticos y didácticos disponibles en diversas fuentes $\mathrm{y}$, por tanto, proporcionan criterios sobre las características deseables de un proceso de instrucción: se pueden utilizar como artefactos de diseño, como recursos metodológicos o como herramienta para evaluar la idoneidad didáctica de procesos de instrucción matemática. Este último, es el fin con el que se aplica la guía que empleamos en el presente estudio.

La GVID-FFE ha sido formulada a partir del análisis de diversas propuestas curriculares y de resultados de investigaciones del campo de la educación matemática-estadística. Es, por tanto, un instrumento válido para valorar procesos de formación estadística en profesores de educación primaria. 


\section{METODOLOGÍA}

La investigación corresponde a un estudio de caso en el que se analiza y evalúa la fase de implementación de un proceso de enseñanza sobre estadística elemental, realizado con un grupo de 58 estudiantes en la Facultad de Educación de la Universidad de Granada (España). El estudio se realizó en dos fases o etapas: (1) descripción del diseño e implementación del proceso de estudio y (2) evaluación de la idoneidad didáctica.

En la primera fase se aplica la técnica de análisis de contenido (cualitativo) para extraer y clasificar HDS y, luego, se utiliza esta información para sintetizar la trayectoria didáctica implementada, teniendo en cuenta las dimensiones epistémica, ecológica, cognitiva, afectiva e instruccional (interaccional y mediacional).

En la segunda fase, para asignar de manera objetiva una valoración al grado de idoneidad, se ha definido como medida relativa el porcentaje de indicadores que se cumplen en cada dimensión, para lo cual se establecen tres niveles: logrado, medianamente logrado y no logrado. De esta forma, es posible identificar la presencia o ausencia de dichos indicadores en cada faceta, y se puede calcular el porcentaje de indicadores cumplidos. Se ha adoptado el siguiente criterio cualitativo de calificación de la idoneidad: si el porcentaje de indicadores cumplidos es menor de 40 se considera baja; si es mayor que 70, alta; si está entre 40 y 70 , media.

\section{DISEÑO DEL PROCESO DE INSTRUCCIÓN}

Se proponen tres proyectos de análisis de datos por medio de los cuales se pretende contextualizar y motivar la emergencia de las nociones y técnicas estadísticas descriptivas elementales (tablas de frecuencias, promedios, dispersiones, comparación de distribuciones de frecuencias) y algunas nociones básicas de probabilidad. Se contempla, además, una colección de ejercicios resueltos del texto Estocástica para Maestros (Batanero y Godino, 2003) y un tablón virtual de docencia como espacio de comunicación asincrónica entre los estudiantes y el profesor. A continuación se presentan los tres proyectos de análisis de datos incluidos en el diseño:

\subsection{PROYECTO 1 (P1): ALUMNO TÍPICO}

Este proyecto se describe con detalle en Batanero y Díaz (2011) y en este caso se ha realizado el siguiente planteamiento, considerando las variables y cuestiones que se indican:

Elaborar un perfil de los alumnos de la clase en las siguientes características (variables estadísticas): género (hombre, mujer); ¿haces deporte? (nada, poco, mucho); número de hermanos (incluyendo al propio estudiante, o sea, número de hijos en la familia); peso (kg); dinero que llevas en el bolsillo (cantidad de euros).

a) ¿Cuáles son las características de un estudiante típico o representativo de la clase?

b) ¿Cómo de representativo es dicho estudiante respecto de la clase?

c) ¿Hay diferencias entre hombres y mujeres en cada una de dichas características? 


\subsection{PROYECTO 2 (P2): LANZAMIENTO DE DOS DADOS}

Se juega con dos dados por parejas. Se lanzan los dados y se suman los puntos obtenidos. Si resulta una suma de 6,7,8, o 9, entonces gana $A$ una ficha; si la suma es distinta de esos números gana $B$ una ficha.

a) ¿Qué prefieres ser: jugador $A$ o $B$ ? Razona la respuesta.

b) ¿Es equitativo este juego? ¿Tiene ventaja un jugador sobre el otro según estas reglas del juego? ¿Quién tiene más probabilidades de ganar? Razona las respuestas.

c) Simula el lanzamiento de dos dados. Juega con un compañero 10 veces y anota los resultados de las sumas que obtienes. ¿Quién ha ganado más veces $A$ o $B$ ? ¿Piensas que se repetirá el resultado si jugamos 100 veces más? ¿Por qué? Razona las respuestas.

Una vez recogidos los datos para el conjunto de las parejas formadas en la clase se plantean las siguientes cuestiones:

d) ¿Quién ha ganado más veces los jugadores $A$ o los $B$ ? ¿Qué ha ocurrido? ¿Por qué no ha ganado más veces $A$ como era de esperar? ¿Qué puede hacer un profesor en esta situación para explicar el resultado a sus alumnos?

e) Construir un diagrama de barras adosadas en el que se represente la distribución de frecuencias relativas y la distribución de probabilidad de la variable aleatoria suma de puntos al lanzar dos dados. ¿Cómo piensas que cambiará este diagrama si en lugar de representar las frecuencias relativas al lanzar 100 veces los dados se hubieran lanzado 10000 veces?

\subsection{PROYECTO 3 (P3): EFICACIA DE UN ENTRENAMIENTO DEPORTIVO}

Un profesor de educación física prepara a un grupo de 60 alumnos de 12 años para participar en una competición. Transcurridos 3 meses del entrenamiento (septiembre a diciembre) quiere comprobar si el entrenamiento ha sido efectivo. Para ello decide comparar el tiempo, en segundos, que los alumnos tardan en recorrer 20 metros en septiembre y en diciembre, y también quiere conocer si hay diferencias entre los chicos y las chicas.

Trabajando en equipo, hay que elaborar un informe para responder razonadamente las cuestiones siguientes, incluyendo los cálculos y gráficos que se consideren pertinentes:

a) ¿Ha sido efectivo el entrenamiento en el conjunto de la clase?

b) ¿Hay diferencias entre chicos y chicas en el tiempo en correr 20 metros inicialmente en septiembre?

c) ¿Hay diferencias entre chicos y chicas en el tiempo en correr 20 metros después del entrenamiento en diciembre?

d) ¿Quién ha mejorado más, los chicos o las chicas?

e) ¿Hay algún alumno (chico o chica) que se pueda considerar como atípico en su capacidad de correr (por su velocidad excesivamente baja)?

f) ¿Qué se debe hacer con los sujetos atípicos desde el punto de vista estadístico? 


\section{DESCRIPCIÓN DE LA TRAYECTORIA DIDÁCTICA IMPLEMENTADA}

El proceso de estudio fue implementado durante dos semanas (correspodiente a 9 horas cronológicas). Durante este tiempo se aplicaron los tres proyectos de análisis de datos y algunas tareas complementarias previstas en el diseño. Lo que sigue corresponde a una síntesis de la trayectoria didáctica implementada. Esta síntesis se ha realizado a partir del análisis de los principales HDS que fueron recogidos durante la implementación de los tres proyectos y está estructurada de acuerdo con las dimensiones epistémica, ecológica, cognitiva, afectiva, interaccional y mediacional.

\subsection{FACETA EPISTÉMICA}

- Los problemas estadísticos (proyectos) son el eje central de la actividad matemáticaestadística. Los estudiantes tienen la oportunidad de formular problemas propios durante la primera clase, pero finalmente no son abordados. Solo se realizan dos tareas de ejercitación relativas a probabilidad.

- Las representaciones de uso convencional (tablas, gráficos, símbolos) de la estadística y la probabilidad son recordadas por el profesor y utilizadas por los estudiantes para resolver los proyectos. Se exploran las potencialidades gráficas que brindan las tecnologías; concretamente, la utilidad de la hoja de cálculo Excel para la construcción de tablas y gráficos, y el uso de programas informáticos para representar la simulación de experimentos aleatorios.

- Se promueven procesos de traducción entre distintas representaciones semióticas; específicamente, se llevan a cabo los siguientes procesos de conversión: (a) en el P1, se representan las características de los individuos como medidas (datos), los datos son representados en tablas y gráficos, y se comunica el significado que surge de los datos en lenguaje natural; (b) en el P2, las sumas obtenidas de lanzar dos dados son representadas en tablas de conteo, los casos posibles se representan en tablas de distribución de probabilidad y las sumas obtenidas en tablas de frecuencias, la distribución de probabilidad y distribución de frecuencias se resume a través de gráficos, y las conjeturas y conclusiones son comunicadas en lenguaje natural; (c) en el P3, los tiempos recorridos en septiembre y diciembre son resumidos en tablas de frecuencias, las distribuciones de frecuencias son representadas mediante gráficos y el significado que emerge de los datos es expresado en leguaje natural.

- Los conceptos y definiciones básicas de la estadística y la probabilidad son recordados por el profesor a través de institucionalizaciones puntuales (dirigidas a algunos estudiantes) y magistrales (dirigidas a toda la clase).

- Los procedimientos fundamentales de la estadística y la probabilidad elemental son empleados por los estudiantes para dar respuesta a las cuestiones planteadas, pero se enfocan principalmente en el cálculo de estadísticos con escasas posibilidades para la construcción de gráficos. 
- Las principales propiedades del tema de estudio son explicadas por el profesor y aplicadas por los estudiantes durante la resolución de los proyectos.

- Los estudiantes recogen datos de encuestas y experimentos (proyecto 1 y 2); sin embargo, estos datos no son utilizados en las etapas posteriores de ambos proyectos.

- Se realizan procesos de comparación basados en datos (comparación de distribuciones de frecuencias correspondientes a muestra de igual y distinto tamaño) y de comparación entre distribución de probabilidad y de frecuencias.

- Se realizan procesos de inferencia (predicciones) en base a la probabilidad; los estudiantes deben aplicar la ley empírica de los grandes números para estimar las frecuencias a partir de la probabilidad de que gane uno u otro jugador (proyecto 2 ).

- Los estudiantes establecen conclusiones basadas en resúmenes estadísticos de los datos (promedios y desviación típica); sin embargo, son muy pocas las oportunidades de realizar conclusiones a partir de representaciones gráficas.

- Se favorece el desarrollo de argumentos basados en datos; en la mayoría de las actividades, se pide a los estudiantes justificar sus respuestas de acuerdo con el resumen estadístico de los datos. Esto se presenta como un contenido difícil para la mayoría de los estudiantes.

- La lectura e interpretación de información estadística presentada en diferentes representaciones (tablas y gráficos) es explicada por el profesor en la mayoría de los casos.

\subsection{FACETA ECOLÓGICA}

- Se realizan conexiones con elementos de la vida cotidiana y con el currículo escolar. Específicamente, en el proyecto 2, se alude al uso de la ley empírica de los grandes números por parte de las compañías de seguro para establecer las primas y se analizan eventuales adaptaciones al proyecto para abordar aprendizajes del currículo escolar.

\subsection{FACETA COGNITIVA}

Se han manifestado como relevantes los siguientes conflictos:

- Proyecto Alumno Típico: comprensión del significado de alumno típico; uso de la media o la moda para determinar el alumno típico en variables cuantitativas discretas; aplicación de la media en lugar de la mediana para determinar el alumno típico en distribuciones asimétricas; uso de una estrategia de conteo en lugar de una técnica estadística para determinar el alumno típico; significado de representatividad de un alumno típico; grado de representatividad de los promedios de acuerdo a la mayor o menor dispersión de los datos (desviación típica); aproximación de la media para determinar el alumno típico en la variable número de hermanos. La media es 2,75, 
¿qué valor tomar 2 o 3?; etiquetado de tablas de frecuencias; elaboración de tablas de frecuencias para variables continúas; tratamiento de variables cualitativas, un estudiante propone el uso de códigos numéricos para representar valores cualitativos y hacer cálculos; persistencia en el uso de la fórmula para calcular la media de datos agrupados en intervalos aun cuando se dispone de medios de cálculos más exactos (calculadora, hoja de cálculo); procedimiento de cálculo de la mediana. Hay estudiantes que tienen dificultades con el manejo de los valores de frecuencia mayor a uno; interpretación del valor máximo de la variable peso, como el valor que se ubica en el bigote derecho del gráfico de caja; no se tienen en cuenta los valores atípicos.

- Proyecto Lanzamiento de dos Dados: los resultados del experimento son equiprobables; dificultad para determinar las sumas posibles y obtener el espacio muestral del experimento; justificación en base al número de valores de la variable aleatoria suma de puntos al lanzar dos dados asociados a cada jugador (en cuatro casos gana A y en siete B), en lugar de considerar las sumas posibles para cada valor; se concluye en base a los resultados obtenidos al simular el lanzamiento de los dados 10 y 100 veces (sesgo de la heurística de representatividad); dificultad para representar las sumas posibles al lanzar dos dados (uso de tabla de doble entrada y diagrama de árbol); dos sumas que aparecen en distinto orden se consideran como un único suceso; dificultad de comprensión con el concepto de distribución de frecuencias (no se reconoce el número de lanzamientos ni se distingue entre el valor de la variable y las frecuencias). En este proyecto se han manifestado, además, varios conflictos relacionados con la construcción e interpretación de gráficos estadísticos, por ejemplo, el uso de frecuencias absolutas en lugar de las frecuencias relativas al comparar las distribución de probabilidad y frecuencias en el gráfico de barras adosadas; también ha habido errores cometidos por futuros profesores de educación primaria en investigaciones previas como en Bruno y Espinel (2005) que nos alertan de la confusión entre histogramas y diagramas de barras, en nuestra investigación se ha manifestado este conflicto en la construcción de todas las barras juntas o todas separadas en el gráfico de barras adosadas; finalmente, un número importante de estudiantes no incluye título en el gráfico ni etiquetas en los ejes, que son elementos estructurales fundamentales de los gráficos estadísticos ya que muestran la información contextual de los mismos, así como las variables representadas y unidades de medida utilizadas (Friel, Curcio y Bright, 2001).

- Proyecto Eficacia de un Entrenamiento Deportivo: comparación sin hacer una reducción estadística de los datos; comparación del mejor tiempo obtenido en septiembre y diciembre; persistencia en el uso de la fórmula en lugar de utilizar la herramienta función de la hoja de cálculo para calcular la media de datos agrupados; dificultad para determinar la efectividad del entrenamiento mediante la media y la desviación típica (no se logran interpretar estos valores); se manifiestan errores en el procedimiento de cálculo de las frecuencias relativas; dificultad en el etiquetado de las columnas de las tablas de frecuencias; se representan en un mismo eje los valores y las marcas de clase; dificultad con el significado, procedimiento de cálculo e interpretación del valor atípico. 


\subsection{FACETA AFECTIVA}

- Las tareas propuestas resultan motivadoras y desafiantes. Destaca la generación de un ambiente de confianza y respeto mutuo en el cual se promueve la comunicación y argumentación en condiciones de igualdad.

\subsection{FACETA INTERACCIONAL}

- La metodología didáctica privilegia las actividades de trabajo en grupos reducidos y las intervenciones magistrales. En las fases de trabajo en grupo se manifiestan diversos conflictos, hay casos en que el profesor sugiere la solución, y rebaja la demanda cognitiva inicial del proyecto (efecto Topaze) (Brousseau, 1986). En las intervenciones magistrales, el profesor anticipa algunos contenidos necesarios para responder las preguntas, y en la fase de institucionalización, si bien da un estatus oficial a la mayoría de los objetos matemáticos no sistematiza ni establece significados claros para algunos conceptos y técnicas estadísticas.

- Se realizan diversas intervenciones evaluativas. En ocasiones el profesor se apoya en las respuestas correctas de algún estudiante, sin considerar los conflictos de aprendizaje de otros.

\subsection{FACETA MEDIACIONAL}

- El tiempo resulta insuficiente para abordar los aprendizajes mediante la metodología propuesta. En el proyecto 1 se opta por trabajar con una matriz de datos previamente recogidos, en lugar de usar los datos recogidos en la clase. En el proyecto 2, en la actividad $d$, se utilizan datos supuestos proporcionados por el profesor. En el proyecto 3 , se entregan las tablas de frecuencias previamente construidas a fin de favorecer la construcción de gráficos.

- Se manifiestan diversos conflictos con el manejo de la hoja de cálculo: uso de la herramienta función, uso de fórmulas, elaboración de tablas de frecuencias y construcción de gráficos.

\section{RESULTADOS. VALORACIÓN DE LA IDONEIDAD DIDÁCTICA}

Como se ha señalado, en el EOS se ha introducido el uso de guías con indicadores empíricos de idoneidad didáctica que permiten caracterizar un proceso de enseñanza-aprendizaje. En la Tabla 1 se indican ejemplos de indicadores aplicados para valorar el proceso formativo descrito. 
Tabla 1. Ejemplos de indicadores de Idoneidad Didáctica

\begin{tabular}{|c|c|}
\hline Faceta/ Componente & Ejemplos de Indicadores \\
\hline \multicolumn{2}{|l|}{ EPISTÉMICA: } \\
\hline Situaciones-problemas & $\begin{array}{l}\text { Se incluye una muestra representativa y articulada de situaciones } \\
\text { problemas (proyectos de análisis de datos) de contextualización, } \\
\text { ejercitación y aplicación. } \\
\text { Se promueve el uso de problemas abiertos que admiten el uso de } \\
\text { estrategias variadas de solución. }\end{array}$ \\
\hline Lenguajes & $\begin{array}{l}\text { Se incluyen diversas representaciones de uso convencional } \\
\text { (representaciones concretas y visuales, tablas, gráficos, } \\
\text { estadísticos, íconos, símbolos) para incentivar su producción por } \\
\text { parte de los alumnos. } \\
\text { Se proponen procesos de traducción entre distintas } \\
\text { representaciones. } \\
\text { Se promueve la capacidad para discriminar entre el uso más } \\
\text { apropiado de distintas representaciones (tablas, gráficos, etc.). }\end{array}$ \\
\hline $\begin{array}{l}\text { Reglas (definiciones, } \\
\text { proposiciones y } \\
\text { procedimientos) }\end{array}$ & $\begin{array}{l}\text { Se incluyen los conceptos, procedimientos y propiedades } \\
\text { fundamentales de la estadística y la probabilidad formulados de } \\
\text { manera correcta. } \\
\text { Se promueven diferentes métodos de recolección de datos } \\
\text { (censos, encuestas, observación, medición, experimentos } \\
\text { aleatorios). }\end{array}$ \\
\hline Argumentos & Se promueve el razonamiento y la demostración. \\
\hline Relaciones & $\begin{array}{l}\text { Los objetos matemáticos-estadísticos (problemas, definiciones, } \\
\text { proposiciones, etc.) se relacionan y conectan entre sí. }\end{array}$ \\
\hline \multicolumn{2}{|l|}{ ECOLÓGICA: } \\
\hline Adaptación al currículo & $\begin{array}{l}\text { Se tienen en cuenta los principios y orientaciones del currículo } \\
\text { escolar. }\end{array}$ \\
\hline $\begin{array}{l}\text { Apertura hacia la innovación } \\
\text { didáctica }\end{array}$ & $\begin{array}{l}\text { Se promueve la innovación basada en la investigación y la } \\
\text { práctica reflexiva. }\end{array}$ \\
\hline $\begin{array}{l}\text { Adaptación social y } \\
\text { profesional }\end{array}$ & $\begin{array}{l}\text { Se establecen conexiones entre los contenidos estadísticos con } \\
\text { diferentes contextos de la vida cotidiana. }\end{array}$ \\
\hline Educación en valores & $\begin{array}{l}\text { Se contempla la formación en valores democráticos y el } \\
\text { pensamiento crítico. }\end{array}$ \\
\hline $\begin{array}{l}\text { Conexiones intra e } \\
\text { interdisciplinares }\end{array}$ & $\begin{array}{l}\text { Se establecen conexiones entre los contenidos estadísticos con } \\
\text { otros contenidos matemáticos y áreas de la formación } \\
\text { universitaria. }\end{array}$ \\
\hline
\end{tabular}




\begin{tabular}{|c|c|}
\hline \multicolumn{2}{|l|}{ COGNITIVA: } \\
\hline Conocimientos previos & $\begin{array}{l}\text { Se conectan los nuevos aprendizajes con los conocimientos } \\
\text { previos, retomando aquellos aprendizajes que los estudiantes no } \\
\text { hayan alcanzado. }\end{array}$ \\
\hline $\begin{array}{l}\text { Adaptaciones curriculares a } \\
\text { las diferencias individuales }\end{array}$ & $\begin{array}{l}\text { Se proponen adaptaciones razonables y apropiadas considerando } \\
\text { las diferencias individuales. } \\
\text { Se incluyen actividades de ampliación y refuerzo. }\end{array}$ \\
\hline $\begin{array}{l}\text { Aprendizaje; evaluación } \\
\text { sumativa }\end{array}$ & Se promueven altos niveles de comprensión y competencia. \\
\hline \multicolumn{2}{|l|}{ AFECTIVA: } \\
\hline Intereses y necesidades & Se tiene en cuenta el carácter motivacional de los problemas. \\
\hline Actitudes & $\begin{array}{l}\text { Se promueve una actitud positiva hacia la materia. } \\
\text { Se favorece la lectura y uso crítico de la información. } \\
\text { Se favorece la argumentación en situaciones de igualdad; el } \\
\text { argumento se valora en sí mismo y no por quién lo dice. }\end{array}$ \\
\hline Emociones & $\begin{array}{l}\text { Se promueve la confianza y seguridad en sí mismo para resolver } \\
\text { tareas matemáticas. }\end{array}$ \\
\hline \multicolumn{2}{|l|}{ INTERACCIONAL: } \\
\hline Interacción docente-discente & $\begin{array}{l}\text { Se tiene en cuenta el uso de diversos recursos retóricos y } \\
\text { argumentativos para implicar y captar la atención de los alumnos. } \\
\text { Se adaptan adecuadamente las lecciones de clase en situaciones } \\
\text { no previstas. }\end{array}$ \\
\hline Interacción entre discentes & Se favorecen instancias de comunicación y debate. \\
\hline Autonomía & Se promueve el trabajo personal de los estudiantes. \\
\hline Evaluación formativa & Se aplican procesos evaluativos de manera sistemática y continua. \\
\hline \multicolumn{2}{|l|}{ MEDIACIONAL: } \\
\hline $\begin{array}{l}\text { Recursos materiales } \\
\text { (tangibles, textuales, } \\
\text { digitales) }\end{array}$ & $\begin{array}{l}\text { Se incorpora el uso de software especializados y recursos de } \\
\text { internet para potenciar los aprendizajes. }\end{array}$ \\
\hline $\begin{array}{l}\text { Número de alumnos, horario } \\
\text { y condiciones del aula }\end{array}$ & $\begin{array}{l}\text { Se tiene en cuenta una organización apropiada del entorno físico } \\
\text { de la clase. } \\
\text { El número de alumnos y su organización es adecuada para llevar } \\
\text { cabo la enseñanza. }\end{array}$ \\
\hline $\begin{array}{l}\text { Tiempo de enseñanza y } \\
\text { aprendizaje (colectiva e } \\
\text { individual) }\end{array}$ & $\begin{array}{l}\text { El tiempo disponible es suficiente para la enseñanza. } \\
\text { Se gestiona el tiempo de manera eficiente. }\end{array}$ \\
\hline
\end{tabular}

Lo que sigue corresponde a la valoración del proceso formativo descrito en los apartados anteriores. En la Tabla 2 se resume el grado de idoneidad didáctica parcial y global y, luego, se realiza un análisis descriptivo relacionando los resultados con los HDS observados durante la implementación. 
Tabla 2. Porcentaje de indicadores cumplidos en cada dimensión

\begin{tabular}{|l|c|c|c|}
\hline \multicolumn{1}{|c|}{ Faceta } & $\begin{array}{c}\mathbf{N}^{\mathbf{0}} \text { de indicadores } \\
\text { posibles }\end{array}$ & $\begin{array}{c}\mathbf{N}^{\mathbf{0}} \text { de indicadores } \\
\text { cumplidos }\end{array}$ & $\begin{array}{c}\text { Porcentaje de } \\
\text { cumplimiento }\end{array}$ \\
\hline Epistémica & 24 & 19 & 79,1 \\
\hline Ecológica & 8 & 4 & 50 \\
\hline Cognitiva & 8 & 5 & 62,5 \\
\hline Afectiva & 9 & 8 & 88,8 \\
\hline Interaccional & 14 & 12 & 85,7 \\
\hline Mediacional & 9 & 6 & 66,6 \\
\hline TOTAL & 72 & 54 & 75 \\
\hline
\end{tabular}

La identificación de los HDS, a partir de los cuales se ha descrito la trayectoria didáctica, estuvo basada en el reconocimiento de un rasgo que determina el grado de idoneidad en alguna de las características del proceso de estudio implementado. Esta particularidad permite analizar los resultados obtenidos en cada dimensión, contrastando los indicadores cumplidos con los HDS que han tenido lugar.

En la faceta epistémica el porcentaje de indicadores cumplidos supera el 70\%, lo que refleja un alto grado de idoneidad, por lo cual destaca como aspecto positivo la alta representatividad de los objetos matemáticos estadísticos puestos en juego. Respecto a los indicadores que no fueron alcanzados (o que se lograron de manera insuficiente), en la componente situaciones problemas, se puede señalar la falta de oportunidades para formular problemas por parte de los estudiantes, y el escaso número de tareas de ampliación y refuerzo. En la componente lenguaje, no se analizaron las ventajas y limitaciones de las representaciones utilizadas para representar la información y tampoco se dieron oportunidades para utilizar representaciones de uso no convencional. Finalmente, en la componente reglas, no se incluyó el estudio de modelos para analizar la relación entre dos variables ni se abordaron procedimientos de selección aleatoria para la obtención de muestras representativas.

En la componente situaciones problemas, el siguiente HDS da cuenta de la única oportunidad que han tenido los estudiantes para generar problemas propios durante el P1. Estos problemas son planteados como posibles variables, pero no llegan a tener una estructura de problema estadístico como tal.

P: [...] En el contexto de este proyecto, ¿en qué otras variables podemos pensar?

E1: Color preferido.

E2: Lleva o no lleva gafas.

E3: La estatura de cada estudiante.

E4: Llevan o no vaqueros.

E5: Color de los ojos.

$\mathrm{P}$ : Bien vemos que es posible elegir distintas variables [...] 
En la dimensión ecológica se observa un nivel de idoneidad media (el porcentaje de indicadores cumplidos está entre el 40 y 70\%). Los indicadores que muestran un alto nivel de cumplimiento refieren a las conexiones establecidas con el currículo escolar y con diferentes contextos de la vida cotidiana; los menos valorados apuntan a la necesidad de conectar la estadística con diferentes profesiones y campos de la ciencia, y con el desarrollo de un espíritu crítico para analizar usos tendenciosos de la información. En la componente conexiones intra e interdisciplinares se requiere, además, establecer conexiones explícitas con otros contenidos matemáticos y con otras disciplinas.

A continuación, se muestran dos HDS que dan cuenta de las conexiones establecidas entre el proceso de estudio con los elementos del entorno durante la realización del P2. Estos HDS permiten explicar los indicadores cumplidos en esta dimensión.

P: [...] Este experimento lo puede hacer un maestro o maestra con estudiantes de primaria. ¿Pensáis que este proyecto podría ser comprensible por los niños de sexto curso?

E1: Sí.

E2: Es muy difícil.

P: Yo creo que sí, lanzar un dado, sumar los puntos, explorar un poco la situación. Fijaros que el tema de casos posibles por casos favorables son reglas que aparecen en los textos de primaria. De modo que este proyecto o una versión más simple, como lanzar un solo dado, pueden ser razonables para trabajar con niños [...].

P: [...] La ley de los grandes números es el fundamento de las compañías de seguros. Las compañías de seguro ¿cómo ponen las primas? Pues a base de recoger estadísticas de accidentes o de otros temas con lo cual tienen distribuciones de frecuencias, pero también tienen unos cálculos probabilísticos, de tal manera que prevén el comportamiento a largo plazo de los fenómenos que están manejando y ajustan la primas para no perder dinero, confiando en que las frecuencias relativas convergerán a las probabilidades $[\ldots]$.

La idoneidad cognitiva ha resultado media. Los indicadores mejor valorados apuntan al uso de los conocimientos previos, la promoción de altos niveles de comprensión y competencia, y las potencialidades del proceso de estudio para poner en evidencia conflictos cognitivos. Una valoración menor ha tenido la inclusión de actividades de ampliación y refuerzo, y el uso de los resultados de las evaluaciones.

El siguiente es un ejemplo de HDS observado en el P2 que muestra la representación errónea del espacio muestral del experimento. Este HDS explica la valoración positiva del proceso de estudio implementado en términos de identificar conflictos cognitivos manifestados en el aprendizaje de los contenidos tratados.

P: [...] Vamos a compartir y discutir las respuestas planteadas a la primera cuestión. Teresa, nos va a explicar cómo han resuelto la tarea en su grupo (Figura 1). Teresa, ¿qué prefieres ser: jugador $\mathrm{A}$ o jugador B? 


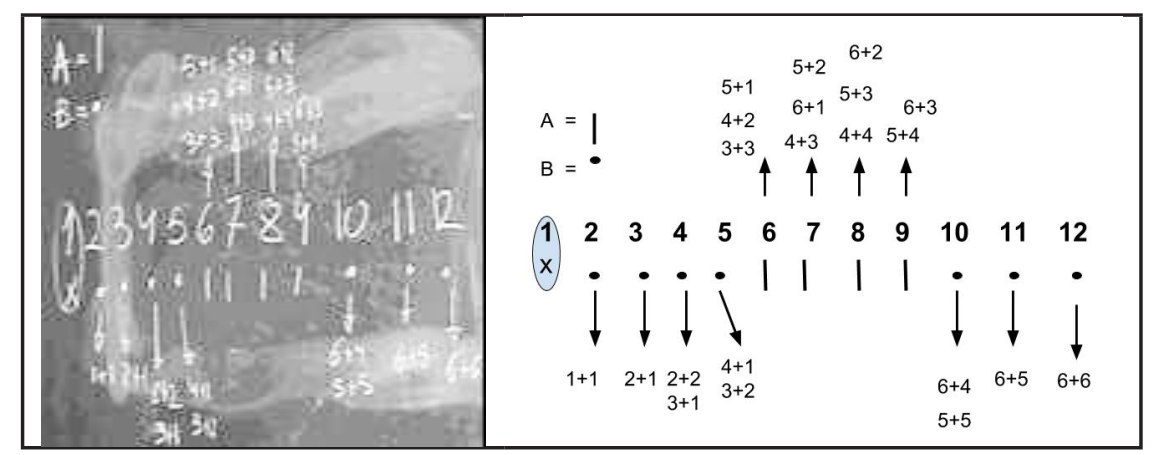

Figura 1. Errores manifestados en el P2

Teresa: Prefiero ser el jugador A.

P: ¿Están de acuerdo los demás? ¿Qué prefieren ser jugador A o B?

E12: No, es mejor ser B.

E13: Yo opino que A.

P: ¿Por qué opinas que es mejor ser B? (se dirige a E12).

E12: Porque de 11 resultados posibles (espacio muestral del experimento), el jugador A puede obtener 6, 7, 8 o 9; entonces, tiene cuatro posibilidades. En cambio, B tiene todas las demás posibilidades, que son siete.

P: ¿Y tú por qué prefieres ser A? (se dirige a Teresa)

Teresa: Porque el jugador A tiene 11 posibilidades, que son las que he anotado arriba. En cambio, B tiene 10, que son las que aparecen abajo.

En este caso E12 justifica su respuesta a partir de los resultados en que gana cada jugador, en lugar de tener en cuenta las sumas posibles para cada resultado (sesgo de la equiprobabilidad) (Lecoutre, 1992); por otra parte, Teresa representa erróneamente las sumas posibles e interpreta dos sumas que aparecen en distinto orden como un único suceso.

A continuación, se presenta un HDS que da cuenta del uso de los resultados de las evaluaciones centrado en las respuestas correctas sin considerar los errores.

P: [...] En la última pregunta se pide construir un diagrama de barras adosadas. Este grupo lo ha hecho bastante bien, ¿por qué les pedía que hicierais un diagrama de barras adosadas y no un histograma? Porque la variable es discreta. Los histogramas son pertinentes para variables continuas y en ese caso las barras están pegadas unas junto a otras [...]. Otro grupo que ha respondido bien es el de Sara, Elena y Sandra. Este grupo ha hecho el gráfico con dos colores; de color verde, está la distribución de frecuencias y de color rosado, la distribución de probabilidad. Se ve como la distribución de probabilidad es muy regular y la distribución de frecuencias no lo es tanto en el resultado de los 100 lanzamientos [...].

En la dimensión afectiva la idoneidad es alta. En esta dimensión, salvo el desarrollo de una actitud crítica sobre la lectura y uso de la información, los demás indicadores se han 
cumplido satisfactoriamente. Si bien esta dimensión es la mejor valorada, sería necesario aplicar un instrumento específico para evaluar los indicadores propuestos de manera individual.

La idoneidad interaccional, al igual que en el caso anterior, es alta. Se cumplieron satisfactoriamente los indicadores referidos a la generación de instancias de comunicación y debate, el uso de variados recursos retóricos para captar el interés de los estudiantes, y la institucionalización de contenidos y resolución de conflictos por parte del profesor. Los indicadores menos valorados fueron los de la componente evaluación formativa; específicamente, los referidos al uso de estrategias y procedimientos variados de evaluación.

Los siguientes HDS, recogidos en los proyectos P1 y P3, dan cuenta de los indicadores cumplidos en esta dimensión. El primero muestra las posibilidades de comunicación y argumentación entre alumnos; y el segundo, la manifestación de un conflicto que motivó la institucionalización de algunos contenidos para facilitar el progreso de los alumnos.

E27: [...] En la pregunta ¿Qué ha ocurrido?, ¿es lo mismo que antes?

E28: No.

E27: ¿Por qué no? Igual ha salido que ha ganado B. Siempre ganará B.

E28: Pero los experimentos son muy pocos [...].

E21: [...] En cuanto a las diferencias, ¿hay que poner todas las diferencias? Es decir; ¿cuántos hombres y mujeres hay?

P: No, es un juicio global del grupo, para hacer ese juicio se puede interpretar la media. E21: ¿Y lo del valor representativo?

P: Se hace interpretando la desviación típica. Vamos a comparar los hombres con las mujeres. ¿La media de las mujeres es más representativa que la media de los hombres en cada uno de sus grupos? Esto se establece en función de la desviación típica, si la desviación típica es mayor es menos representativa.

En la dimensión mediacional, la idoneidad es media. Los indicadores que presentaron una valoración favorable son los referidos a la integración de recursos y la gestión del tiempo; los que presentaron una valoración menor, corresponden al número de alumnos y el tiempo disponible para la enseñanza.

Se muestra un HDS observado en el P2 que nota la integración de un Apple con el que se simuló el lanzamiento de dos dados.

P: [...] Se ha simulado aquí el lanzamiento de los dados 100 y 10.000 veces (Figura 2). Así se ve el comportamiento de la suma, en último caso la forma que tiene la frecuencia relativa es prácticamente igual que la distribución de probabilidad [...]

Finalmente, la valoración global del proceso de estudio ha resultado alta. No obstante, habrá que tener en cuenta las dificultades específicas señaladas y las limitaciones de los instrumentos para recoger evidencias el trabajo individual. 

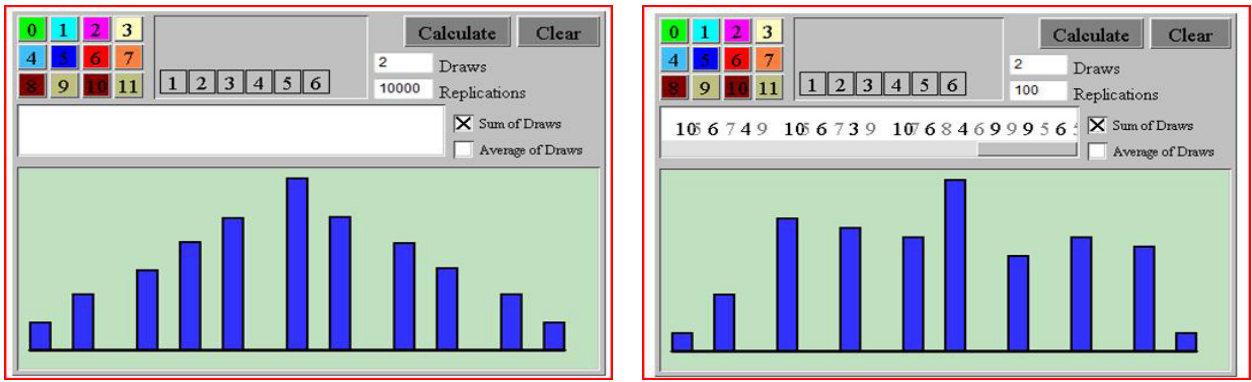

Figura 2. Simulación del lanzamiento de dos dados

\section{DISCUSIÓN Y CONSIDERACIONES FINALES}

Se ha constatado que es posible contextualizar los conocimientos estadísticos elementales mediante el uso de proyectos realistas de análisis de datos. La resolución de los proyectos Alumno Típico y Eficacia de un Entrenamiento Deportivo han permitido motivar el uso de los principales conceptos y técnicas de la estadística descriptiva. Asimismo, el proyecto Lanzamiento de dos Dados incorpora las nociones probabilísticas básicas. Esta metodología didáctica ha permitido implementar fases de trabajo indagativo, colaborativo y dialógico entre el profesor y los estudiantes, que permiten identificar y resolver conflictos de aprendizaje a lo largo de la trayectoria didáctica.

La valoración de las idoneidades parciales y el contraste con los HDS ha permitido conocer ventajas y limitaciones del proceso formativo implementado. Desde el punto de vista epistémico, las situaciones problemas elegidas han permitido contextualizar la mayoría de los objetos matemáticos-estadísticos del conocimiento común y avanzado que un profesor de educación primaria debe manejar; se requerirá prestar atención a la formulación de problemas por parte de los estudiantes y la inclusión de contenidos que permitan el estudio de la relación entre dos variables y de la inferencia estadística elemental e intuitiva (representatividad de una muestra, estimación de parámetros). En la dimensión ecológica, se establecieron conexiones adecuadas con elementos socioculturales y con el currículo escolar, es necesario potenciar las relaciones intra e interdisciplinares del currículo universitario. En la dimensión cognitiva, si bien los resultados muestran información relevante, es necesario incluir nuevos instrumentos que permitan complementar la información recogida; específicamente, se requiere tener evidencias sobre la progresión del aprendizaje para describir con mayor precisión el desarrollo de la trayectoria cognitiva. Una situación similar vislumbra en la dimensión afectiva, en la cual el grado de idoneidad demanda tener en cuenta las actitudes y creencias que se manifiestan de manera individual. Finalmente, en la dimensión instruccional (interaccional y mediacional), se destacan como aspectos positivos los formatos de interacción y la integración de recursos; como aspectos a mejorar, están los procesos de evaluación formativa y el tiempo asignado para desarrollar la actividad.

Es propio de las investigaciones orientadas al diseño instruccional la aplicación de varios ciclos de investigación, mediante los cuales los medios y estrategias que se diseñan e implementan se van mejorando progresivamente. En cierto modo, la investigación realizada, 
en un primer ciclo, puede no ser del todo adecuada. El análisis de la implementación, junto a otros ciclos de carácter retrospectivo, resulta esencial para introducir cambios fundamentados en los sucesivos ciclos, que permitan progresivamente obtener diseños e implementaciones más idóneas y ajustadas a las restricciones normativas.

\section{REFERENCIAS BIBLIOGRÁFICAS}

Batanero, C., Burrill, G. \& Reading, C. (Eds.) (2011). Teaching statistics in school mathematicschallenges for teaching and teacher education: A joint ICMI/IASE study. Berlin: Springer Science+Business Media. DOI: 10.1007/978-94-007-1131-0

Batanero, C. y Díaz, C. (2004). El papel de los proyectos en la enseñanza y aprendizaje de la estadística. En J. Patricio (Ed.), Aspectos didácticos de las matemáticas (pp. 125-164). Zaragoza: ICE.

Batanero, C. y Díaz, C. (Eds.) (2011). Estadística con proyectos. Granada: Universidad de Granada. Recuperado el 15 de junio de 2016 desde, http://www.ugr.es/ batanero/pages/librostesis.html.

Batanero, C. y Godino J. D. (2003). Estocástica y su didáctica para maestros. Granada: Universidad de Granada. Recuperado el 12 de mayo de 2017 desde, http://www.ugr.es/ jgodino/edumatmaestros/welcome.htm.

Brousseau, G. (1986). Fondements et méthodes de la didactique des mathématiques. Recherches en Didactique des Mathématiques, 7(2), 33-115.

Bruno, A. y Espinel, M. (2005). Recta numérica, escalas y gráficas estadísticas: un estudio con estudiantes para profesores. Formación del Profesorado e Investigación en Educación Matemáticas, 7, 57-85.

Franklin, C., Kader, G., Mewborn, D., Moreno, J., Peck, R., Perry, M. \& Scheaffer, R. (2005). Guidelines for assessment and instruction in statistics education (GAISE) report: A Pre-K-12 curriculum framework. United States of America: American Statistical Association. Recuperado el 31 de julio de 2017 de http://www.amstat.org/Education/gaise/GAISEPreK-12_Full.pdf

Friel, S., Curcio, F. \& Bright, G. (2001). Making sense of graphs: critical factors influencing comprehension and instructional implications. Journal for Research in mathematics Education, 32(2), 124-158. DOI: $10.2307 / 749671$

Godino, J. (2013). Indicadores de la idoneidad didáctica de procesos de enseñanza y aprendizaje de las matemáticas. Cuadernos de Investigación y Formación en Educación Matemática, 11, $111-132$.

Godino, J., Batanero, C. \& Font, V. (2007). The onto-semiotic approach to research in mathematics education, The International Journal on Mathematics Education, 39(1), 127-135.

Godino, J., Contreras, A. y Font, V. (2006). Análisis de procesos de instrucción basado en el enfoque ontológico-semiótico de la cognición matemática, Recherches en Didactiques des Mathématiques, 26(1), 39-88.

Godino, J., Rivas, H., Arteaga, P., Lasa, A. y Wilhelmi, M. (2014). Ingeniería didáctica basada en el enfoque ontológico-semiótico del conocimiento y la instrucción matemáticos. Recherches en Didactique des Mathématiques, 34(2-3), 167-200.

Lecoutre, M. P. (1992). Cognitive models and problem spaces in "purely random" situations. Educational Studies in Mathematics, 23, 557-568.

Ministerio de Educación y Ciencia (2006). Real Decreto 1631/2006, de 29 de diciembre, por el que se establecen las enseñanzas mínimas correspondientes a la Educación Secundaria Obligatoria. Madrid: MEC.

Ministerio de Educación Cultura y Deporte (2014). Real Decreto 126/2014, de 28 de febrero, por el que se establece el currículo básico de la Educación Primaria. Madrid: MECD.

National Council of Teachers of Mathematics (2000). Principios y estándares para la educación 
matemática (Manuel Fernández, trad.). Sevilla: Sociedad Andaluza de Educación Matemática Thales. (Trabajo original publicado en 2000).

National Council of Teachers of Mathematics (2014). Principles to actions: Ensuring mathematical success for all. Reston: NCTM.

Rivas, H. (2014). Idoneidad didáctica de procesos de formación estadística de profesores de educación primaria. (Tesis doctoral). Universidad de Granada. Granada.

Wilhelmi, M., Font, V. y Godino, J. (2005). Bases empíricas de modelos teóricos en didáctica de las matemáticas: Reflexiones sobre la Teoría de Situaciones Didácticas y el Enfoque Ontológico y Semiótico. En: Colloque International «Didactiques: quelles references epistemologiques. Recuperado el 5 de marzo de 2017 de http://www.ugr.es/ jgodino/funciones-semioticas/bases_ empiricas_5junio06.pdf 
